\title{
Variable pressure cornual resection. Does radical hysteroscopic surgery give better results?
}

Received: 22 July 2005 / Accepted: 29 November 2005 / Published online: 22 July 2006

(C) Springer-Verlag Berlin / Heidelberg 2006

\begin{abstract}
The radicality of the resective technique at transcervical endometrial resection (TCRE) may affect both the safety and efficacy. We evaluated both a more and a less extreme technique and compared results with our standard TCRE. A nonrandomised, prospective cohort study of three standardised endometrial resection/ablation techniques, each with differing tissue destruction profiles, was performed on 270 patients. Percentage reduction in bleeding, satisfaction, complications, and failures were recorded. Group 1 had standard loop resection circumferentially from the fundus to the internal os with current blend 1, power $120 / 60 \mathrm{~W}$. The method for group 2 was the same as for group 1, with additional radical cornual resection followed by rollerball ablation to the entire cavity and cervical canal using the same power settings. To establish the safety of the cornual resection component, 25 cases were performed under laparoscopic control. In the first 15 cases, the extra cornual material excised was weighed separately and its contribution to the total weight calculated. Group $3 \mathrm{had}$ slow low-power rollerball ablation to the cavity, cornua, and cervical canal with unmodulated power at $30 \mathrm{~W}$. There were 68,131 , and 71 patients in the respective groups, followed up for a maximum of 36 months, with amenorrhoea rates of $31 \%, 36 \%$, and $31 \%$. Failure (a need for a further procedure) occurred in $18 \%, 12 \%$, and $20 \%$,
\end{abstract}

J. T. M. Clark $(\bowtie) \cdot$ L. Dupuch

Royal Devon and Exeter Hospital,

Gladstone Road, Heavitree,

Exeter, EX12ED, UK

e-mail: James.Clark@rdehc-tr.swest.nhs.uk

I. Balchin

Imperial College,

London, UK

D. L. Byrne

Royal Cornwall Hospital,

Treliske, Truro,

Cornwall, UK

J. M. Frappell

Derriford Hospital,

Plymouth, Devon, UK respectively, and the preoperative to postoperative percentage reduction in bleeding between groups 2 and 3 was $78 \%$ (standard deviation 26) and 79\% (standard deviation 33). There were no statistical differences in clinical outcome measures, complications, or satisfaction rates. The extra cornual endometrium comprised a mean additional $9 \%$. This study did not support the premise that more radical techniques confer advantage in clinical outcome, although a trend towards a lower failure rate with the radical resection method may achieve significance with greater numbers. Principally, the greater failure rate in the rollerball group was due to cyclical pain despite improvement in bleeding score. Where satisfaction and safety is consistently high, the most straightforward procedure of rollerball ablation may be preferable, but the possible higher failure rate with this technique will require further qualification.

Keywords Endometrial resection - Rollerball ablation · Amenorrhoea

\section{Introduction}

Endometrial resection has been evaluated in many centres and has been shown to be an acceptable alternative to medical management [1] or hysterectomy [2-5]. Amenorrhoea rates, however, vary between $30 \%$ and $60 \%$, with the best results obtained almost exclusively from centres most proficient in these techniques [6].

If amenorrhoea is to be the chief goal, total and permanent destruction or removal of all endometrial tissue should be the objective. Continuation of bleeding immediately after resection would suggest persisting functional endometrial tissue. This can be explained either by lack of operator skill or by use of a technique that incompletely covers the uterine cavity, leaving, for example, the endocervical canal and uterine cornua. It has been proposed that the cornual segment of the fallopian tube represents a danger area susceptible to perforation [7]; regardless, it forms an area of the cavity that is also difficult to reach surgically without an effective technique. 
There is also histologic evidence of endometrial gland survival regardless of power settings after endometrial ablation [8] and of both focal and diffuse endometrial regeneration over time following complete endometrial destruction [9-12]. These findings may explain cyclical pain in amenorrhoeic women and the resumption of menstruation following a bleed-free interval.

Previous work has identified how increasing the radicality of the resection technique may improve outcome without compromising safety [13]. In a series of 927 patients at up to 5 years the failure rate at endometrial resection was reduced from $22 \%$ to $13 \%$ by the addition of rollerball ablation to the cavity following resection (Table 1). A further reduction in failure rate to $5 \%$ was achieved when additional radical resection of the fundus and cornua was performed under laparoscopic control, with a concomitant increase in the amenorrhoea rate from $47 \%$ to $70 \%$.

The purpose of our study was to prospectively test the efficacy of a technique of combined radical resection and ablation that included a variable glycine pressure method to effectively remove endometrial tissue lying within the cornua, and to compare this with the results achieved after the standard method of TCRE. We hoped to develop a reproducible method of radical resection that would achieve the excellent outcomes demonstrated in Browne's series [13] but that could be safely performed without the need for laparoscopic control. The technique was also evaluated for safety, and in addition, the extra tissue removed was calculated. The purpose of this was to explore the premise that low amenorrhoea rates are due to the persistence of functional endometrial tissue after endometrial resection. If a significant amount of extra material was removed, then a better result might be expected in the short term, and also a potential focus for endometrial regeneration in the longer term would have been eliminated.

\section{Additional study component: rollerball ablation alone}

The original intention had been to evaluate radical resection compared with standard resection only. By the time 131 patients had undergone the procedure using method 2, the first 45 had reached 3 years since the operation and were evaluated as a preliminary group. Because initial results suggested that the radical resection appeared not to

Table 1 Results from Browne [13] (TCRE transcervical endometrial resection)

\begin{tabular}{llll}
\hline & Amenorrhoea & Failure & Number \\
\hline Technique 1 (TCRE) & $47 \%$ & $22 \%$ & 238 \\
Technique 2 & $50 \%$ & $13 \%$ & 470 \\
(TCRE + rollerball) & & & \\
Technique 3 & $70 \%$ & $5 \%$ & 219 \\
(TCRE + rollerball + & & & \\
further fundal and & & & \\
cornual resection) & & & \\
\hline
\end{tabular}

confer advantage in terms of efficacy outcome, and although complications had not been a problem, it was felt more useful to continue the study comparing methods 1 and 2 with the more straightforward procedure of rollerball ablation.

\section{Methods}

This was a nonrandomised trial, with the different cohorts of patients evaluated sequentially in three different units. All patients were operated on by a single surgeon, with standardised techniques and identical equipment. At initiation of the radical resection study, 68 (71\%) patients had replied to their 3-year outcome questionnaire after TCRE, and these data were used for baseline comparisons (group 1).

\section{Group 1: standard resection (TCRE)}

Sixty-eight women who had undergone endometrial resection were evaluated at 3 years from their operation dates. The procedures were performed by a single surgeon utilising a standardised technique. All women suffered menorrhagia refractory to a trial of medical treatment using tranexamic acid and mefenamic acid. Other options, including the Mirena intrauterine system and hysterectomy, had been discussed. All women received a single dose of Prostap $3.75 \mathrm{mg}$ (Wyeth) or Zoladex $3.6 \mathrm{mg}$ (AstraZeneca) 5 weeks prior to the procedure, which was performed on a day-case list unless anaesthetic protocols required an overnight stay.

Total intravenous anaesthesia was induced, and the patient placed in lithotomy position. The bladder was emptied, and a vulsellum was placed on the anterior lip of the cervix, which was dilated to Hegar 10. A standard size resectoscope utilising a cutting loop with diathermy set at cut $120 \mathrm{~W}$, coagulate $60 \mathrm{~W}$ on blend 1 was used to circumferentially resect the endometrium in strips from the fundus to the internal os with glycine flow produced via a Unifusor pressure bag set at $200 \mathrm{~mm} \mathrm{H}_{2} \mathrm{O}$. The cavity was resected to a depth that showed the linear white fibres of myometrium. Depending on the degree of endometrial preparation, this might require repeated passes. Disposable reservoir drapes allowed accurate and continuous calculation of glycine fluid balance and absorption.

\section{Group 2: radical resection}

The technique was initially performed as in group 1 . Following this, the cornua were resected separately by a variable pressure method as follows (Fig. 1): The cutting loop was rotated laterally and located just distal to the cornu. The suction tap and glycine inflow tap were then both fully opened to give continuous flow with removal of distension pressure. This collapsed the cavity, and as the cornu "winked" shut, the resectoscope was swung in a deep loop, thus excising a cone-shaped sample of tissue con- 
Fig. 1 a The resection loop is positioned adjacent to the right cornu. b Suction is activated to collapse the cavity, and the cornu "winks" shut. c After the resection cut, suction is turned off to expand the cavity and show residual endometrium within cornu requiring a second cut. d Cornual chip resulting from the first cut
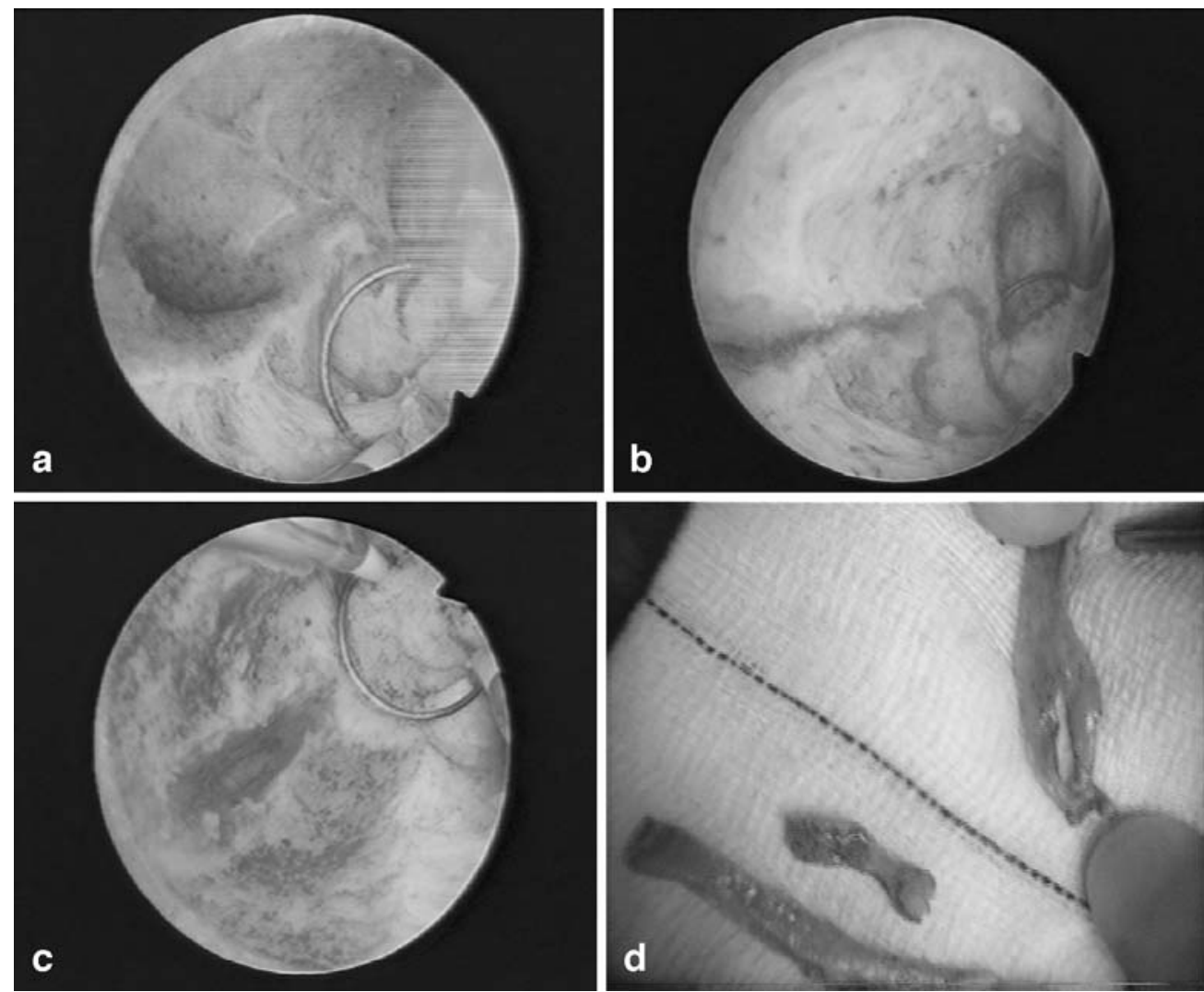

taining the endometrium within the cornu. If endometrial tissue was seen remaining, a second pass was performed to excise deeper in a top-hat arrangement. The opposite cornu was then removed in the same way.

The cornua were resected at the end of the procedure because only after the majority of the cavity is treated does the uterus become compliant enough to safely collapse and distend under total control of the glycine pressure as regulated by the inflow. We called this procedure variable pressure cornual resection (VPCR). Following the resection, the cutting loop was replaced by a rollerball and the cavity ablated in entirety, including the endocervical canal and cornua, using the same power settings.

Patients undergoing the radical resection (RR) technique (group 2) were initially overseen by the surgeon who developed the VPCR component. This procedure had been used over a number of years without incident. Nevertheless, for the purpose of demonstrable safety, the first 25 patients were operated on under laparoscopic control. To meet local ethics committee approval, these patients were those also listed for a concomitant laparoscopic sterilisation to avoid an additional and nonclinically indicated procedure. For these patients, two laparoscopic stacks were required.

For the first 15 procedures, additional endometrial material resected from the cornua was weighed separately and the results expressed as a percentage of the whole. These were the chips from within the cornual area that would have remained unless the VPCR method had been used. The endometrium within the endocervical canal was treated by ablation, rather than resection, as this area is susceptible to bleeding that is difficult to deal with because the continuous-flow mechanism of the resectoscope funcions improperly so close to the external os.

Group 3: additional study component—rollerball ablation alone

There were 71 patients who underwent the more straightforward technique of rollerball ablation (RA), performed with a very slow roll at $30 \mathrm{~W}$ with unmodulated (pure cutting) current. Unmodulated current was used to give the greatest depth of tissue necrosis and avoid an eschar that would inhibit further electrical conduction [8]. The cornua and endocervical canal were also treated. Later in the series, a new resectoscope was purchased that utilised a larger rollerball. On advice from the hospital medical physics department, the current used was increased to $70 \mathrm{~W}$ to give the same current density as $30 \mathrm{~W}$ with the smaller ball.

\section{Outcome measures and data collection}

Surgical time, fluid balance of the distension medium, complications, and presence of fibroids were recorded. The presence of histologic evidence of adenomyosis was also documented. 
Table 2 Patients' subjective assessment of number of days of menstruation per month

\begin{tabular}{llllll}
\hline Menses & Score $(\mathrm{S})$ & Number of days $(\mathrm{N}) /$ month & Total preop $(\mathrm{S}) \times(\mathrm{N})$ & Total postop & Percent reduction \\
\hline Spotting & 1 & & & \\
Light & 2 & & & \\
Moderate & 3 & & & \\
Heavy & 4 & A & $\mathrm{B}$ & $\mathrm{R}^{\mathrm{a}}=(\mathrm{A}-\mathrm{B}) / \mathrm{A} \times 100$ \\
Flood & 5 & & & \\
Bleeding score & sum $(\mathrm{S} 1 \times \mathrm{N} 1)+(\mathrm{S} 2 \times \mathrm{N} 2)$, etc.
\end{tabular}

${ }^{\mathrm{a}} \mathrm{R}=$ percentage reduction in bleeding score

Following their procedures, patients were discharged home the same evening with open access to the gynaecology ward. Patients were then reviewed in the nurse-led outpatient clinic at 3 months and then subsequently by telephone interview or postal questionnaire.

The primary outcome was amenorrhoea rate, as defined by absolutely no vaginal bleeding from 3 months after the procedure to the time of final follow-up. Satisfaction rates were recorded on the basis of a quality-of-life questionnaire directed at menstrual symptoms, including whether the patient would recommend the operation to a friend. The failure rate involved patients requiring any further procedure for menstrual dysfunction, such as Mirena, repeat TCRE, or hysterectomy.

For patients within the main body of the study (methods 2 and 3), pre- and postprocedure menstrual assessment was more exacting. Secondary outcome measures included percentage reduction in bleeding score and improvement in dysmenorrhoea, as well as the need for further surgery and the satisfaction rate. The bleeding score was derived from the sum of the number of days of bleeding per month, each scored by degree of heaviness. This was recorded by the patient on a menstrual chart over 3 months (Table 2). Although the bleeding score was a subjective measurement, because patients provided a second bleeding score at follow-up they acted as their own controls, and the percentage change therefore represented an objective calculation.

Table 3 Main results at study end: 36 months for groups 1 and 2 and 24 months for group 3 (TCRE transcervical endometrial resection, $R R$ radical resection, $R A$ rollerball ablation)

\begin{tabular}{ccccl}
\hline & Amenorrhoea & Failures & Satisfaction & $\begin{array}{l}\text { Percent } \\
\text { reduction } \\
\text { in bleeding } \\
\text { score }\end{array}$ \\
\hline $\begin{array}{l}\text { Method 1 } \\
\text { (TCRE) }\end{array}$ & $21 / 6831 \%$ & $12 / 6818 \%$ & $49 / 6872 \%$ & N/A \\
$\begin{array}{c}\text { Method 2 } \\
\text { (RR) }\end{array}$ & $47 / 13136 \%$ & $16 / 131 \quad 12 \%$ & $95 / 13173 \%$ & $78 \%$ \\
$\begin{array}{c}\text { Method 3 } \\
\text { (RA) }\end{array}$ & $22 / 7131 \%$ & $14 / 7120 \%$ & $53 / 7175 \%$ & $79 \%$ \\
\hline
\end{tabular}

Follow-up was to be at 3 years. Local ethics committee permission was obtained, and statistical analysis was performed by the Research and Development Support Unit at the Royal Devon and Exeter Hospital.

\section{Results}

\section{Group 1}

Three-year data were available on $71 \%$ of the patients in group 1 (TCRE).

In this group, $31 \%$ of women reported amenorrhoea, with $18 \%$ having undergone or being listed for another procedure such as repeat resection or hysterectomy. Overall, $72 \%$ of patients described themselves as satisfied with the result and would recommend the procedure to a friend (Table 3). There were no perforations or documented episodes of glycine overload $>11$ in this group.

\section{Group 2}

Data were complete on 131 patients in the radical resection group, representing a return rate of $88 \%$ of patients who had reached 3 years of follow-up. This excluded two amenorrhoeic patients. One had had histology reporting

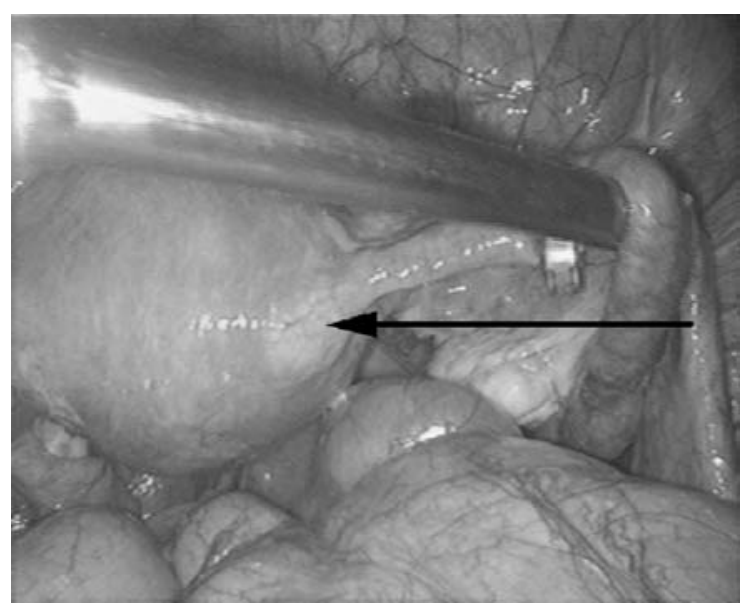

Fig. 2 Laparoscopic view during variable pressure resection of right cornu. Note the serosal blanching 
Table 4 Main results for all groups at annual follow-ups ( $A$ amenorrhoea rate, $F$ failure rate/need for another procedure, $S$ satisfaction rate, $T C R E$ transcervical endometrial resection, $R R$ radical resection, $R A$ rollerball ablation)

\begin{tabular}{|c|c|c|c|c|c|c|c|c|c|c|}
\hline & Number & A & $\mathrm{F}$ & $\mathrm{S}$ & A & $\mathrm{F}$ & $\mathrm{S}$ & A & $\mathrm{F}$ & $\mathrm{S}$ \\
\hline Follow-up & & \multicolumn{3}{|c|}{12 months } & \multicolumn{3}{|c|}{24 months } & \multicolumn{3}{|c|}{36 months } \\
\hline Method 1 (TCRE) & 68 & & & & & & & $31 \%$ & $18 \%$ & $72 \%$ \\
\hline Method 2 (RR) & 131 & $60 \%$ & $5 \%$ & $91 \%$ & $45 \%$ & $9 \%$ & $81 \%$ & $36 \%$ & $12 \%$ & $73 \%$ \\
\hline Method 3 (RA) & 71 & $39 \%$ & $16 \%$ & $76 \%$ & $31 \%$ & $20 \%$ & $75 \%$ & & & \\
\hline
\end{tabular}

"atypical complex glandular hyperplasia," and the other had "appearances suggestive of well-differentiated endometrioid endometrial adenocarcinoma." Both patients subsequently underwent hysterectomy, and in both cases histology was normal in all sections. The original slides were also reviewed without change in diagnosis.

Forty-seven patients $(36 \%)$ reported total amenorrhoea, and the calculated mean reduction in pre- to postoperative bleeding score was $78 \%$. Twelve percent of patients required a second procedure (failures), and $73 \%$ described satisfaction with their result and would recommend the operation to a friend (Table 3). One patient required a laparoscopy for possible perforation after sudden loss of glycine to the 1-1 maximum was noted towards the end of the procedure. The laparoscopy was normal, and the patient remained amenorrhoeic for 2 years but subsequently needed a hysterectomy for resumption of light but painful menses.

All 25 patients who had procedures performed under laparoscopic control were noted to have blanching of the cornual area (Fig. 2), but no loss of structural integrity was noted, nor was any loss of glycine into the peritoneal cavity observed. No patients within the study had evidence of bowel or organ damage or signs suggestive of transmitted thermal energy to the bowel.

The extra endometrial chippings taken separately from the first 15 patients represented an extra mean $1.04 \mathrm{~g}(8.9 \%$ of total) material by weight.

There was a trend in favour of a reduced failure rate in group 2 (RR) vs. group 1(TCRE) that failed to reach statistical significance $(\chi 2 P=0.29)$, and a similar amenorrhoea rate $(36 \%$ vs. $31 \% ; \chi 2 P=$ not significant).

\section{Group 3}

Complete data were available on 71 group 3 (RA) women at 2 years $(95 \%)$.

Twenty-two women (31\%) had complete amenorrhoea, their mean reduction in bleeding score was $79 \%$, and there were 14 failures $(20 \%$; Table 3$)$. The increased failure rate compared with the $12 \%$ for the radical resection group was not statistically significant $(\chi 2 P=0.15)$. There were no incidents of fluid overload or haemorrhage in this group. One perforation occurred at initial dilatation, the part of the technique that was generic for all three methods. The satisfaction rate was $75 \%$.

\section{Discussion}

The weakness of this study lies in its nonrandomised nature; however, the large study size and good return rate provide reassurance as to the safety of all three techniques, including that of the cornual resection when performed by the VPCR method. All techniques were safe in the day-case setting, and the one perforation occurred during dilatation, a procedure generic to all three operation methods.

Satisfaction rates with operation outcome were all above $70 \%$, in line with the published literature [2-5]. The procedures were all effective, with the objective, patientderived bleeding reduction scores approaching $80 \%$ in groups 2 and 3 and amenorrhoea rates all between $31 \%$ and $36 \%$.

The study also suggests that the most technically straightforward method of rollerball ablation was statistically as effective as the more radical resection technique. This is based on the lack of significance in the $\chi 2$ evaluation of an increased failure rate from $12 \%$ to $20 \%$. If correct, this finding supports the use of rollerball ablation, which has previously been described as the safest of the first-generation procedures [7] and is simpler and quicker to learn. However, greater numbers and longer follow-up may demonstrate significant improvement with the more radical technique, and a true $20 \%$ failure rate with rollerball ablation may be unacceptably high for many patients exercising the choice of conservative surgery over hysterectomy.

Differing trends are seen when comparing the annual outcomes for the different groups throughout the study. This information was available for methods 2 and 3 (Table 4).

The 12-month evaluation of radical resection showed an amenorrhoea rate of $60 \%$, falling to $45 \%$ at 24 months and

Table 5 Bleeding scores for groups 2 and 3 with predominant reason for failure ( $R R$ radical resection, $R A$ rollerball ablation)

\begin{tabular}{lll}
\hline & $\begin{array}{l}\text { Group 2 } \\
\text { (RR) }\end{array}$ & $\begin{array}{l}\text { Group 3 } \\
\text { (RA) }\end{array}$ \\
\hline Number & 131 & 71 \\
Response rate complete data & $88 \%$ & $95 \%$ \\
Mean reduction bleeding score & $77.896 \%$ & $79.302 \%$ \\
Amenorrhoea rate & $35.88 \%$ & $30.99 \%$ \\
Failure rate & $12.21 \%$ & $19.72 \%$ \\
Mean reduction bleeding score & $33.691 \%$ & $53.738 \%$ \\
$\quad$ in failures & & \\
Pain as predominant cause & $17.65 \%$ & $71.42 \%$ \\
$\quad$ of failure & & \\
\hline
\end{tabular}


to $36 \%$ at 3 years, with a similar linear increase in failure rate from $5 \%$ to $9 \%$ and finally to $12 \%$. The failures in this group were predominantly due to resumption of heavy menses, with the mean reduction in bleeding score for this subgroup (group 2 failures) being 34\%, and only $17.65 \%$ recording cyclical pain as the reason for failure (Table 5).

Group 3 patients (RA) at 12 months who had achieved amenorrhoea (39\%) had a lasting effect, with the rate a little lower at 24 months $(31 \%)$. The cause of failure in this group was predominately due to the development of cyclical pain (71.42\%) and occurred early, as suggested by the $16 \%(n=7)$ failure rate at 12 months, falling only minimally to $20 \%$ at 2 years. This may be explained by the published findings of surviving endometrial glands beneath the destruction zone after endometrial rollerball ablation, regardless of power settings [12]. The percentage reduction in bleeding score was better in this subgroup (group 3 failures) at $53.74 \%$, despite the increased failure rate compared with group 2.

This study does not confirm Browne's findings, nor does it answer the question of which technique is most effective. Although improved outcome was demonstrated with the more radical technique, this failed to achieve statistical significance with the 270 patients within this study. The failure rate of $12-20 \%$ approximates that of a recent paper comparing 5-year follow-up of microwave endometrial ablation and TCRE, recording hysterectomy rates of $16 \%$ and $25 \%$, respectively, but with higher 5-year amenorrhoea rates of $65 \%$ and $69 \%$ [14]. Such excellent results with a second-generation technique may suggest a questionable future for first-generation procedures.

We believe, however, that within each unit at least one surgeon should remain proficient in endometrial resection in order to provide choice and to manage the large, congenitally abnormal or scarred uterus and patients with submucous fibroids or Asherman's syndrome. In skilled hands, the outcomes from first-generation methods should remain acceptably high, but it is likely that these procedures will become part of more specialised practice only. The joint BSGE/RCOG Special Skills Module in Hysteroscopic Surgery could become an entry requirement for those wishing to embark on training in complex hysteroscopic procedures.

In summary, this study confirms that in practiced and proficient hands, the VPCR/ablation technique is a safe and effective day-case procedure. It indicates an approximate $88 \%$ chance of providing sufficient resolution of menstrual symptoms to avoid hysterectomy at least 3 years from surgery and suggests that radical resection may be the superior of the techniques evaluated in this series.

\section{References}

1. Cooper KG, Parkin DE, Garratt AM, Grant AM (1997) A randomised comparison of medical and hysteroscopic management in women consulting a gynaecologist for treatment of heavy menstrual loss. Br J Obstet Gynaecol 104(12): $1360-1366$

2. Lethaby A, Shepperd S, Cooke I, Farquhar C (2000) Endometrial resection and ablation versus hysterectomy for heavy menstrual bleeding. Cochrane Database Syst Rev 2:CD000329

3. Alexander DA, Naji AA, Pinion SB, Mollison J, Kitchener HC, Parkin DE, et al (1996) Randomised trial comparing hysterectomy with endometrial ablation for dysfunctional uterine bleeding: psychiatric and psychosocial aspects. BMJ 312 (7026):280-284

4. O'Connor H, Broadbent JA, Magos AL, McPherson K (1997) Medical Research Council randomised trial of endometrial resection versus hysterectomy in management of menorrhagia. Lancet 349(9056):897-901

5. Rogerson L, Hawe J, Duffy S (2000) Modern approaches to management of menorrhagia. Hosp Med 61(2):90-92

6. Garry R (2002) Evidence and techniques in endometrial ablation: consensus. Gynaecol Endosc 11(1):5-17

7. Overton C, Hargreaves J, Maresh M (1997) A national survey of the complications of endometrial destruction for menstrual disorders: the MISTLETOE study. Minimally Invasive Surgical Techniques-Laser, EndoThermal or Endorescetion. Br J Obstet Gynaecol 104(12):1351-1359

8. Onbargi LC, Hayden R, Valle RF, Del Priore G (1993) Effects of power and electrical current density variations in an in vitro endometrial ablation model. Obstet Gynecol 82(6):912-918

9. Seeras RC, Gilliland GB (1997) Resumption of menstruation after amenorrhea in women treated by endometrial ablation and myometrial resection. J Am Assoc Gynecol Laparosc 4(3): 305-309

10. Davis JR, Maynard KK, Brainard CP, Purdon TF, Sibley MA, King DD (1998) Effects of thermal endometrial ablation. Clinicopathologic correlations. Am J Clin Pathol 109(1):96-100

11. Taskin O, Onoglu A, Inal M, Turan E, Sadik S, Vardar E et al (2002) Long-term histopathologic and morphologic changes after thermal endometrial ablation. J Am Assoc Gynecol Laparosc 9(2):186-190

12. Letterie GS, Hibbert ML, Britton BA (1993) Endometrial histology after electrocoagulation using different power settings. Fertil Steril 60(4):647-651

13. Browne DS (1996) Endometrial resection-a comparison of techniques. Aust N Z J Obstet Gynaecol 36(4):448-452

14. Cooper KG, Bain C, Lawrie L, Parkin DE (2005) A randomised comparison of microwave endometrial ablation with transcervical resection of the endometrium; follow up at a minimum of five years. BJOG 112(4):470-475 